\title{
New records of lignocellulolytic basidiomycetes (Fungi): Parque Estadual da Serra do Tabuleiro (P.E.S.T.), Santa Catarina, Brazil
}

\author{
Clarice Loguercio-Leite* \\ Josué Michels \\ Juliano Marcon Baltazar \\ Laboratório de Micologia, Departamento de Botânica, Centro de Ciências Biológicas Universidade Federal de Santa Catarina, \\ CEP 88010-970, Florianópolis - SC, Brazil \\ *Author for correspondence \\ clleite@ccb.ufsc.br
}

Submetido em 15/03/2008

Aceito para publicação em 24/05/2008

\begin{abstract}
In a recent survey on lignolicolous basidiomycetes in the State Park of Serra do Tabuleiro (Santa Catarina, Brazil), 27 previously unrecorded species of polypores were collected, namely Antrodia albida, Ceriporiopsis pannocincta, Coriolopsis rigida, Fomitiporia flavomarginata, F. punctata, F. undulata, Fuscoporia callimorpha, F. ferrea, F. punctatiformis, Hexagonia hydnoides, Junghuhnia undigera, Megasporoporia cavernulosa, M. setulosa, Pachykytospora papyracea, Perenniporia medulla-panis, P. ohiensis, P. stipitata, Phellinus allardii, P. bambusarum, P. cesatii, P. melanodermus, Phylloporia chrysita, Polyporus leprieurii, Rigidoporus lineatus, Schizopora flavipora, S. paradoxa and Trametes socotrana.
\end{abstract}

Key words: Basidiomycota, diversity, rain forest

\section{Resumo}

Novas citações de basidiomicetes lignocelulolíticos para o Parque Estadual da Serra do Tabuleiro (P.E.S.T.), SC, Brasil. Um levantamento recente da micota de basidiomicetes lignocelulolíticos no Parque Estadual da Serra do Tabuleiro resultou em 27 espécies inéditas para esta área, a saber: Antrodia albida, Ceriporiopsis pannocincta, Coriolopsis rigida, Fomitiporia flavomarginata, F. punctata, F. undulata, Fuscoporia callimorpha, F. ferrea, F. punctatiformis, Hexagonia hydnoides, Junghuhnia undigera, Megasporoporia cavernulosa, M. setulosa, Pachykytospora papyracea, Perenniporia medulla-panis, P. ohiensis, P. stipitata, Phellinus allardii, P. bambusarum, P. cesatii, P. melanodermus, Phylloporia chrysita, Polyporus leprieurii, Rigidoporus lineatus, Schizopora flavipora, S. paradoxa e Trametes socotrana.

Unitermos: Basidiomycota, diversidade, floresta atlântica 


\section{Introduction}

The white- and brown-rot caused by basidiomycetes, which is a highly variable process, mainly depends upon decomposer organisms and plant species, and also on the micro-habitat (Blanchette, 1995). The high level of biological diversity is very important in tropical forests (Gaston, 2000; Gilbert et al., 2002; Suryanarayanan et al., 2003). Even though these habitats cover only $7 \%$ of the Earth's surface, they represent more than $50 \%$ of species from a biota whose destruction has been accelerated in recent years. Deforestation is therefore achieving the extinction of hundreds of thousands of plant species and all associated species, as well as lignocellulolytic basidiomycetes (Myers, 1997; Wilson, 1997; Sippola et al., 2001).

In the State of Santa Catarina (26 $06^{\circ}$ ' to $30^{\circ} 00^{\prime}$ 'S lat, $48^{\circ} 30^{\prime}$ to $54^{\circ} 00^{\prime} \mathrm{W}$ long), there are two state parks, Serra Furada and Serra do Tabuleiro (Parque Estadual da Serra do Tabuleiro, P.E.S.T.). Serra do Tabuleiro is the largest (nearly $90,000 \mathrm{ha}$, about $1 \%$ of the total state area). The mainland area of the park encompasses part of Águas Mornas, Florianópolis, Garopaba, Imaruí, Palhoça, Paulo Lopes, Santo Amaro da Imperatriz, São Bonifácio and São Martinho municipalities.

The park has a wide diversity of relief and habitats, which permits the protection of an unestimatable biological diversity that has become one of the most representative remnants of the Atlantic Rain Forest of Southern Brazil (Zambonim, 2001).

A former lignolitic basidiomycetes inventory carried out at P.E.S.T. registered the presence of 47 species, 14 of which were the first records from Santa Catarina State (Groposo and Loguercio-Leite, 2004).

This study was proposed in order to expand knowledge about the diversity of wood rot basidiomycetes (Basidiomycota) at P.E.S.T.

\section{Materials and Methods}

Periodical fungal collections were carried out between 2004 and 2005, at 17 sample sites, through random hike/trekking, by conventional procedures
(Guerrero and Homrich, 1999). For identification, the macro- and micromorphological analyses of the specimens were prepared according to Singer (1975) and Ryvarden and Johansen (1980). Distribution data were obtained from the following sources: Fidalgo (1968); Bononi et al. (1981); Bononi (1982); Ryvarden (1987); Rajchenberg and Meijer (1990); Loguercio-Leite and Wright (1991); Silveira and Guerrero (1991); Almeida Filho et al. (1993); Coelho (1994); Gugliotta and Capelari (1995); Jesus (1996); Gerber and LoguercioLeite (1997 and 2000); Soares and Gugliotta (1998); Fonseca (1999); Gerber et al. (1999); Góes-Neto (1999); Gonçalves and Loguercio-Leite (2001); Groposo and Loguercio-Leite (2002); Ryvarden and Meijer (2002); Gilbertoni and Cavalcanti (2003); Sótão et al. (2003); Gilbertoni et al. (2004).

Vouchers were deposited after examination in the FLOR Herbarium (Holmgren et al., 1990). Taxonomic arrangements followed Kirk et al. (2001).

\section{Results and Discussion}

These species, recorded for the first time from P.E.S.T., are also new records from the municipalities, except Florianópolis.

\section{Hapalopilaceae Jülich}

Ceriporiopsis pannocincta (Romell) Gilb. \& Ryvarden, Mycotaxon 22(2): 364, 1985.

Description: Gilbertson \& Ryvarden, 1986.

Distribution: cosmopolitan; Brazil (Santa Catarina).

Material examined: Brazil, Santa Catarina, São Martinho, Vargem do Cedro, Michels \& MarconBaltazar 302, 19///2005 (FLOR); ibid, Águas Mornas, Michels 406, 18/II/2005 (FLOR).

\section{Hymenochaetaceae Imazeki \& Toki}

Fomitiporia flavomarginata Murrill, N. Amer. Fl. 9(1): 11, 1907.

Description: Loguercio-Leite \& Wright, 1995. 


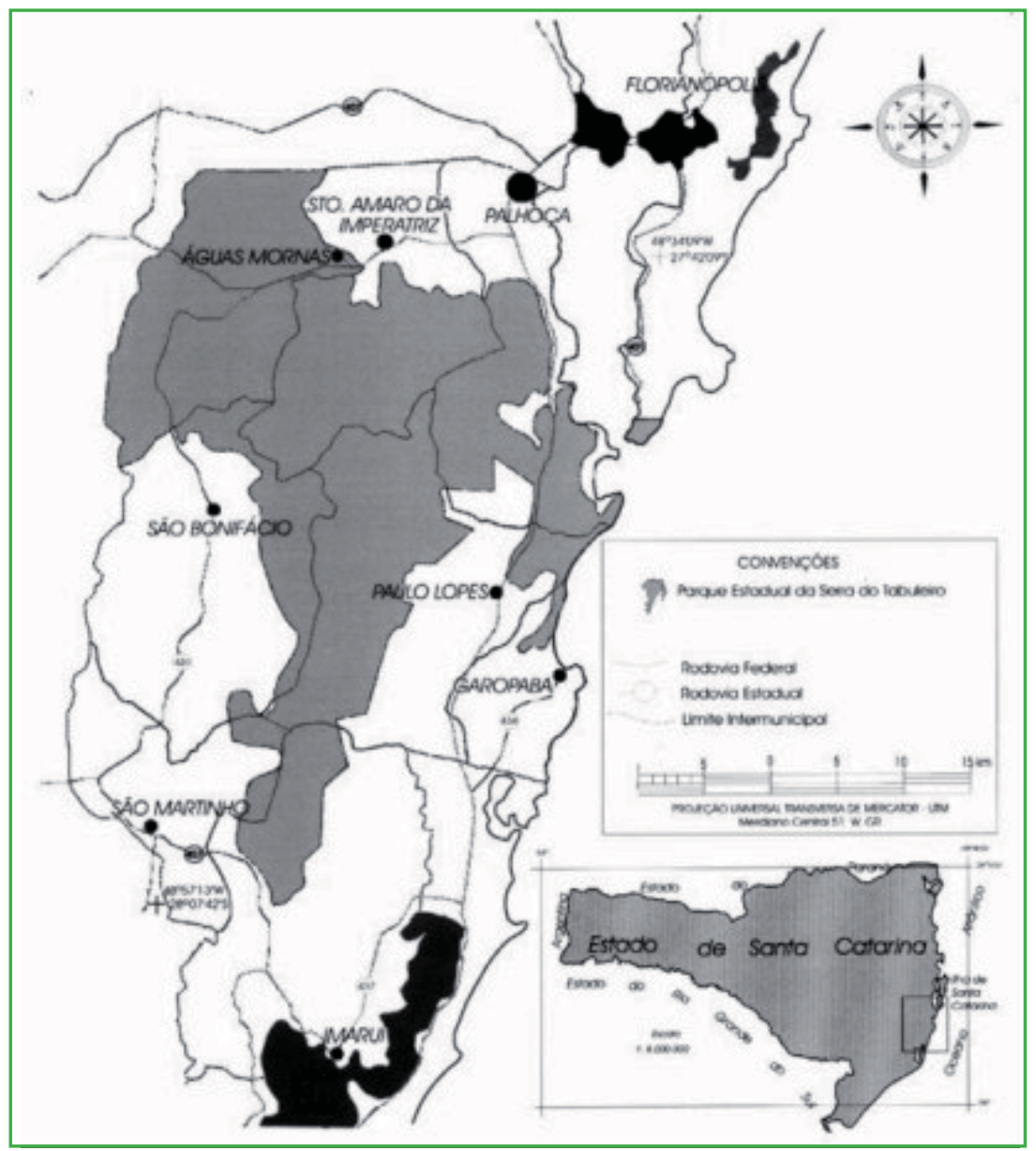

FIGURE 1: Map of Parque Estadual da Serra do Tabuleiro (P.E.S.T.), located within municipalities, Santa Catarina, Brazil (Source: Mapa Político do Estado de Santa Catarina. Final design: Ricardo Drechsler-Santos).

Distribution: neotropical; Brazil (Santa Catarina).

Material examined: Brazil, Santa Catarina, Santo Amaro da Imperatriz, Trilha da Cascata, Michels \& Marcon-Baltazar 010, 17/IV/2004 (FLOR).

Fomitiporia punctata (Fr.) Murrill, Lloydia 10: 254, 1947.

Description: Loguercio-Leite \& Wright, 1995.

Distribution: cosmopolitan; Brazil (Amapá, Paraná, Santa Catarina and São Paulo).

Material examined: Brazil, Santa Catarina, Santo Amaro da Imperatriz, Trilha da Cascata, Michels \& Marcon-Baltazar 066, 05/VI/2004 (FLOR); ibid, Paulo Lopes, Albardão, Michels, Esber \& Marcon-Baltazar 084, 086, 04/IX/2004 (FLOR); ibid, Paulo Lopes,
Albardão, Michels, Esber \& Marcon-Baltazar 115, 05/ IX/2004 (FLOR); ibid, Paulo Lopes, Espraiado, Michels, Klier \& Marcon-Baltazar 147, 10/X/2004 (FLOR); ibid, Paulo Lopes, Laranjal, Michels \& Esber 208, 27/XI/2004 (FLOR); ibid, São Bonifácio, Michels, Santos e Tortato 222, 17/XII/2004 (FLOR); ibid, Águas Mornas, ipse 292, 18/I/2005 (FLOR); ibid, São Martinho, Vargem do Cedro, ipse 337, 19/I/2005 (FLOR); ibid, Imaruí, ipse 364, 372, 20/I/2005 (FLOR ); ibid, Imaruí, ipse 384, 20/I/2005 (FLOR); ibid, Santo Amaro da Imperatriz, Michels, Esber, Groposo \& Marcon-Baltazar 465, 20/III/2005 (FLOR); ibid, Palhoça, Michels, Klier \& Marcon-Baltazar 518, 524, 15/V/2005 (FLOR).

Fomitiporia undulata Murrill, N. Amer. Fl. 9(1): 10, 1907.

Description: Loguercio-Leite \& Wright, 1995. 
Distribution: neotropical; Brazil (Alagoas and Santa Catarina).

Material examined: Brazil, Santa Catarina, Águas Mornas, Michels \& Marcon-Baltazar 252, 17/I/2005 (FLOR); ibid, Imaruí, ipse 393, 20/I/2005 (FLOR).

Fuscoporia callimorpha (Lév.) Groposo, C.L.Leite \& Góes-Neto, Mycotaxon 101: 57, 2007.

Description: Loguercio-Leite \& Wright, 1995.

Distribution: pantropical; Brazil (Paraná, Santa Catarina and São Paulo).

Material examined: Brazil, Santa Catarina, São Bonifácio, Michels, Santos \& Tortato 228, 17/XII/2004 (FLOR).

Fuscoporia ferrea (Pers.) G. Cunn., Bull. N.Z. Dept. Sci. Industr. Res. Pl. Dis. Div. 73: 7, 1948.

Description: Loguercio-Leite \& Wright, 1995.

Distribution: cosmopolitan; Brazil Rio Grande do Sul, Santa Catarina and São Paulo.

Material examined: Brazil, Santa Catarina, Águas Mornas, Michels e Marcon-Baltazar 244, 17/I/2005 (FLOR); ibid, Águas Mornas, ipse 263, 18/I/2005 (FLOR); ibid, Imaruí, ipse 357, 360, 20/I/2005 (FLOR); ibid, Imaruí, ipse 379, 20/I/2005 (FLOR); ibid, Santo Amaro da Imperatriz, Morro das Três Voltas, Michels, Esber, Groposo \& Marcon-Baltazar 478, 20/III/2005 (FLOR); ibid, Palhoça, Michels, Klier \& MarconBaltazar 527, 528, 15/V/2005 (FLOR).

Fuscoporia punctatiformis (Murrill) Zmitr., Malysheva \& Spirin, Nov. Sist. Niz. Rast. 40: 185, 2006.

Description: Loguercio-Leite \& Wright, 1995.

Distribution: neotropical; Brazil (Rio Grande do Sul and Santa Catarina).

Material examined: Brazil, Santa Catarina, Palhoça, Michels, Klier e Marcon-Baltazar 519, 15/ V/2005 (FLOR).

Phellinus allardii (Bres.) S. Ahmad, Basidiomyc. W. Pakist.: 57, 1972.

Description: Larsen \& Cobb-Poulle, 1990.
Distribution: pantropical; Brazil (Santa Catarina).

Material examined: Brazil, Santa Catarina, Santo Amaro da Imperatriz, Trilha da Cascata, Michels \& Marcon-Baltazar 056, 057, 065, 05/VI/2004 (FLOR).

Phellinus bambusarum (Rick) M.J. Larsen, Syn. Fungorum 3: 40, 1990.

Description: Larsen \& Cobb-Poulle, 1990.

Distribution: neotropical; Brazil (Rio Grande do Sul, Paraná and Santa Catarina.

Material examined: Brazil, Santa Catarina, Águas Mornas, Michels \& Marcon-Baltazar 237, 240, 17/I/2005 (FLOR); ibid, ipse, 275, 288, 18/I/2005 (FLOR); ibid, Michels 415, 420, 18/II/2005 (FLOR).

Phellinus cesatii (Bres.) Ryvarden, Norweg. J. Bot. 19: 234, 1972.

Description: Loguercio-Leite \& Wright, 1995.

Distribution: pantropical; Brazil (Santa Catarina).

Materiais examined: Brazil, Santa Catarina, Santo Amaro da Imperatriz, Trilha da Cascata, Michels \& Marcon-Baltazar 047, 05/VI/2004 (FLOR); ibid, Águas Mornas, ipse 239, 251, 17/I/2005 (FLOR); ibid, Águas Mornas, ipse 279, 18/I/2005 (FLOR); ibid, Águas Mornas, Michels 402, 18/II/2005 (FLOR).

Phellinus melanodermus (Pat.) M. Fidalgo, Mem. New York Bot. Gard. 17:135, 1968.

Description: Ryvarden \& Johansen, 1980.

Distribution: pantropical; Brazil (Santa Catarina).

Material examined: Brazil, Santa Catarina, Águas Mornas, Michels 410, 18/II/2005 (FLOR).

Phylloporia chrysita (Berk.) Ryvarden, Norweg. J. Bot. 19: 235, 1972.

Description: Ryvarden, 2004.

Distribution: pantropical; Brazil (Bahia, Paraná, Pernambuco, Rio Grande do Sul and Santa Catarina). 
Material examined: Brazil, Santa Catarina, Santo Amaro da Imperatriz, Trilha da Cascata, Michels \& Marcon-Baltazar 014, 017, 17/IV/2004 (FLOR); ibid, Santo Amaro da Imperatriz, Trilha da Cascata, ipse 033, 18/IV/2004 (FLOR).

\section{Meripilaceae Jülich}

Antrodia albida (Fr.) Donk, Persoonia 4(3): 339, 1966.

Description: Ryvarden \& Johansen, 1980.

Distribution: cosmopolitan; Brazil (Paraná, Santa Catarina and São Paulo).

Material examined: Brazil, Santa Catarina, Paulo Lopes, Laranjal, Michels \& Esber 166, 169, 26/XI/2004 (FLOR); ibid, Imaruí, Michels \& Marcon-Baltazar 477, 20/I/2005 (FLOR); ibid, Palhoça, Michels, Klier \& Marcon-Baltazar 499, 503, 506, 15/V/2005 (FLOR).

Rigidoporus lineatus (Pers.) Ryvarden, Norweg. J. Bot. 19: 236, 1972.

Description: Gilbertson and Ryvarden, 1987.

Distribution: pantropical; Brazil (Alagoas, Amapá, Bahia, Paraíba, Paraná, Pernambuco, Rio Grande do Sul, Santa Catarina and São Paulo).

Material examined: Brazil, Santa Catarina, Santo Amaro da Imperatriz, Trilha da Cascata, Michels \& Marcon-Baltazar 015, 17/IV/2004 (FLOR); ibid, Santo Amaro da Imperatriz, Trilha da Cascata, ipse 036, 18/ IV/2004 (FLOR); ibid, Paulo Lopes, Laranjal, Michels e Esber 210, 27/XI/2004 (FLOR); ibid, São Bonifácio, Michels, Santos \& Tortato 226, 17/XII/2004 (FLOR); ibid, Águas Mornas, ipse 248, 258, 17/I/2005 (FLOR); ibid, Águas Mornas, ipse 269, 18/I/2005 (FLOR); ibid, São Martinho, Vargem do Cedro, ipse 301, 325, 347, 19/I/2005 (FLOR); ibid, Santo Amaro da Imperatriz, Morro das Três Voltas, Michels, Esber, Groposo \& Marcon-Baltazar 481, 487, 20/III/2005 (FLOR).

\section{Polyporaceae Fr. ex Corda}

Coriolopsis rigida (Berk. \& Mont.) Murrill, N. Amer. Fl. 9(2): 75, 1908.

Description: Gilbertson \& Ryvarden, 1986.
Distribution: neotropical; Brazil (Alagoas, Pará, Paraíba, Paraná, Pernambuco, Rio Grande do Sul, Roraima, Santa Catarina, São Paulo and Sergipe).

Material examined: Brazil, Santa Catarina, Palhoça, Sede do P.E.S.T., Michels, Dreschler-Santos \& Marcon-Baltazar 006, 13/III/2004 (FLOR); ibid, Paulo Lopes, Laranjal, Michels \& Esber 152, 161, 167, 26/ XI/2004 (FLOR); ibid, Paulo Lopes, Laranjal, Michels \& Esber 181, 212, 27/XI/2004 (FLOR); ibid, São Martinho, Vargem do Cedro, Michels \& Marcon-Baltazar 318, 333, 343, 19/I/2005 (FLOR ); ibid, Águas Mornas, Michels 417, 18/II/2005 (FLOR); ibid, Palhoça, Michels, Klier \& Marcon-Baltazar 501, 505, 15/V/2005 (FLOR); ibid, Palhoça, Michels, Klier \& Marcon-Baltazar 582, 583, 16/V/2005 (FLOR).

Hexagonia hydnoides (Sw.) M. Fidalgo, Mem. New York Bot. Gard.17: 64, 1968.

Description: Ryvarden \& Johansen, 1980.

Distribution: pantropical; Brazil (Alagoas, Bahia, Mato Grosso, Pará, Paraíba, Paraná, Pernambuco, Santa Catarina, São Paulo and Sergipe).

Material examined: Brazil, Santa Catarina, Palhoça, Sede do P.E.S.T, Michels, Dreschler-Santos \& Marcon-Baltazar 009, 13/III/2004 (FLOR); ibid, São Martinho, Vargem do Cedro, Michels \& MarconBaltazar 305, 19/I/2005 (FLOR); ibid, Águas Mornas, Michels 441, 443, 446, 18/II/2005 (FLOR); ibid, Palhoça, Michels, Klier \& Marcon-Baltazar 507, 513, 15/V/2005 (FLOR); ibid, Palhoça, Michels, Klier \& Marcon-Baltazar 539, 555, 567, 16/V/2005 (FLOR).

Megasporoporia cavernulosa (Berk.) Ryvarden, Mycotaxon 16(1): 174, 1982.

Description: Ryvarden et al., 1982.

Distribution: pantropical; Brazil (Alagoas, Paraíba, Paraná, Pernambuco, Rio Grande do Norte, Santa Catarina, São Paulo and Sergipe).

Material examined: Brazil, Santa Catarina, Paulo Lopes, Laranjal, Michels \& Esber 201, 27/XI/2004 (FLOR); ibid, São Bonifácio, Michels, Santos \& Tortato 225, 17/XII/2004 (FLOR); ibid, Águas Mornas, Michels 431, 440, 18/II/2005 (FLOR); ibid, Santo Amaro da 
Imperatriz, Morro das Três Voltas, Michels, Esber, Groposo \& Marcon-Baltazar 486, 20/III/2005 (FLOR); ibid, Palhoça, Michels, Klier \& Marcon-Baltazar 530, 15/V/2005 (FLOR).

Megasporoporia setulosa (Henn.) Rajchenb., Mycotaxon 16(1): 174, 1982.

Description: Ryvarden et al., 1982.

Distribution: pantropical; Brazil (Paraná and Santa Catarina).

Material examined: Brazil, Santa Catarina, Paulo Lopes, Albardão, Michels, Esber \& Marcon-Baltazar 114, 05/IX/2004 (FLOR); ibid, Paulo Lopes, Espraiado, Michels, Klier \& Marcon-Baltazar 128, 10/X/2004 (FLOR); ibid, Paulo Lopes, Laranjal, Michels \& Esber 180, 191, 193, 27/XI/2004 (FLOR); ibid, Águas Mornas, Michels \& Marcon-Baltazar 284, 297, 18/I/2005 (FLOR); ibid, São Martinho, Vargem do Cedro, Michels \& Marcon-Baltazar 312, 317, 19/I/2005 (FLOR).

Pachykytospora papyracea (Schwein.) Ryvarden, Norweg. J. Bot. 19: 233, 1972.

Description: Gilbertson \& Ryvarden, 1987.

Distribution: neotropical; Brazil (Santa Catarina).

Material examined: Brazil, Santa Catarina, Paulo Lopes, Laranjal, Michels \& Esber 177, 188, 27/XI/2004 (FLOR); ibid, Águas Mornas, Michels 413, sobre Bambusa spp, 18/II/2005 (FLOR).

Perenniporia medulla-panis (Jacq.) Donk, Persoonia 5(1): 76, 1967.

Description: Gilbertson \& Ryvarden, 1987.

Distribution: cosmopolitan; Brazil (Bahia, Paraná, Santa Catarina, São Paulo and Sergipe).

Material examined: Brazil, Santa Catarina, Águas Mornas, Michels \& Marcon-Baltazar 233, 17///2005 (FLOR); ibid, São Martinho, Vargem do Cedro, ipse 328, 19///2005 (FLOR).

Perenniporia ohiensis (Berk.) Ryvarden, Norweg. J. Bot. 19: 143, 1972.

Description: Gilbertson \& Ryvarden, 1987.

Distribution: neotropical; Brazil (Bahia and Santa Catarina).
Material examined: Brazil, Santa Catarina, Imaruí, Michels \& Marcon-Baltazar 388, 20/I/2005 (FLOR); ibid, Santo Amaro da Imperatriz, Morro das Três Voltas, Michels, Esber, Groposo \& Marcon-Baltazar 477, 20/III/2005 (FLOR).

Perenniporia stipitata Ryvarden, Mycotaxon 28(2): 535, 1987.

Description; Ryvarden, 1987.

Distribution: neotropical; Brazil (Rondônia and Santa Catarina).

Material examined: Brazil, Santa Catarina, Águas Mornas, Michels 409, 18/II/2005 (FLOR); ibid, Palhoça, Michels, Klier \& Marcon-Baltazar 520, 15/V/2005 (FLOR).

Polyporus leprieurii Mont., Annls Sci. Nat., Bot., sér. 2 13: 203, 1840.

Description: Nunez \& Ryvarden, 1995.

Distribution: neotropical; Brazil (Alagoas, Bahia, Mato Grosso, Paraíba, Paraná, Pernambuco, Rio Grande do Sul, Santa Catarina, São Paulo and Sergipe).

Material examined: Brazil, Santa Catarina, São Bonifácio, Michels, Santos \& Tortato 227, 17/XII/2004 (FLOR).

Trametes socotrana Cooke, Grevillea 11: 39, 1882.

Description: Gilbertson \& Ryvarden, 1987.

Distribution: pantropical; Brazil (Santa Catarina).

Material examined: Brazil, Santa Catarina, Paulo Lopes, Laranjal, Michels \& Esber 172, 26/XI/2004 (FLOR).

\section{Schizoporaceae Jülich}

Schizopora flavipora (Berk. \& M.A. Curt. ex Cooke) Ryvarden, Mycotaxon 23: 186, 1985.

Description: Gilbertson \& Ryvarden, 1987.

Distribution: cosmopolitan; Brazil (Alagoas, Paraíba, Paraná, Rio Grande do Norte and Santa Catarina). 
Material examined: Brazil, Santa Catarina, Águas Mornas, Michels \& Marcon-Baltazar 272, 18/I/2005 (FLOR); ibid, São Martinho, Vargem do Cedro, ipse 335, 19/I/2005 (FLOR); ibid, Imaruí, ipse 391, 20/I/2005 (FLOR); ibid, Palhoça, Michels, Klier \& Marcon-Baltazar 510, 15/V/2005 (FLOR); ibid, Palhoça, Michels, Klier \& Marcon-Baltazar 561, 16/V/2005 (FLOR).

Schizopora paradoxa (Schrad.) Donk, Persoonia 5(1): 76, 1967.

Description: Gilbertson \& Ryvarden, 1987.

Distribution: cosmopolitan; Brazil (Alagoas, Paraíba, Paraná, Pernambuco, Santa Catarina and São Paulo).

Material examined: Brazil, Santa Catarina, Paulo Lopes, Laranjal, Michels \& Esber 187, 27/XI/2004 (FLOR); ibid, Águas Mornas, Michels \& MarconBaltazar 232, 17/I/2005 (FLOR); ibid, São Martinho, Vargem do Cedro, Michels \& Marcon-Baltazar 308, 19/I/2005 (FLOR); ibid, Águas Mornas, Michels 433, 436, 18/II/2005 (FLOR).

\section{Steccherinaceae Parmasto}

Junghuhnia undigera (Berk. \& M.A. Curtis) Ryvarden, Mycotaxon 20(2): 359, 1984.

Description: Ryvarden, 1984.

Distribution: neotropical; Brazil (Pará, Paraná, Rio Grande do Sul, Santa Catarina and São Paulo.

Material examined: Brazil, Santa Catarina, Santo Amaro da Imperatriz, Trilha da Cascata, Michels \& Marcon-Baltazar 079, 05/VI/2004 (FLOR); ibid, Águas Mornas, ipse 253, 256, 17/I/2005 (FLOR); ibid, São Martinho, Vargem do Cedro, ipse 311, 19/I/2005 (FLOR); ibid, Santo Amaro da Imperatriz, Morro das Três Voltas, Michels, Esber, Groposo \& Marcon-Baltazar 491, 20/III/2005 (FLOR); ibid, Palhoça, Michels, Klier \& Marcon-Baltazar 563, 16/V/2005 (FLOR).

In conclusion, this survey added 27 species of lignocellulolytic basidiomycetes (Basidiomycota) to a previous inventory, with 47 species (Groposo and Loguercio-Leite, 2004). Therefore, the number of species recorded to date from the P.E.S.T. totals 74 .
The geographical distribution shows, for the majority of all species identified, a tropical pattern, so some of them are pantropical (Fuscoporia callimorpha, Hexagonia hydnoides, Megasporoporia cavernulosa, $M$. setulosa, Phellinus allardii, P. cesatii, P. melanodermus, Phylloporia chrysita, Rigidoporus lineatus and Trametes socotrana), while others are neotropical (Coriolopsis rigida, Fomitiporia flavomarginata, F. undulata, Fuscoporia punctatiformis, Junghuhnia undigera, Pachykytospora papyraceae, Perenniporia ohiensis, P. stipitata, Phellinus bambusarum and Polyporus lepreurii).

On the other hand, the minority are cosmopolitan (Antrodia albida, Ceriporiopsis pannocincta, Fomitiporia punctata, Fuscoporia ferrea, Perenniporia medullapanis, Schizopora flavipora and S. paradoxa).

All of them are wood white-rots, except Antrodia albida, which is a brown rot.

\section{Acknowledgements}

The authors are especially grateful to the reviewers for their help in the correction and improvement of the present article.

\section{References}

Almeida Filho, O. M.; Bueno, R.; Bononi, V. L. R. 1993. Algumas espécies de fungos basidiomicetos dos manguezais do estado de São Paulo. Hoehnea, 20: 87-92.

Blanchette, R. A. 1995. Degradation of the lignocellulose complex in wood. Canadian Journal of Botany, 73: 999-1010.

Bononi, V. L. R. 1982. Fungos macroscópicos de Rio Branco, Acre, Brasil. Hoehnea, 19: 31-37.

Bononi, V. L. R.; Trufem, S. F. B.; Grandi, R. A. P. 1981. Fungos macroscópicos do Parque Estadual das Fontes do Ipiranga, São Paulo, Brasil, depositados no herbário do Instituto de Botânica. Rickia, 9: 37-53.

Coelho, G. 1994. Himenoquetáceas com poros (Basidiomycetes) do limite sul da Serra Geral em Santa Maria, RS. Master's Thesis, Universidade Federal do Rio Grande do Sul, Brasil, 112pp.

Fidalgo, M. E. P. K. 1968. Contribution to the fungi of Mato Grosso, Brazil. Rickia, 3: 171-219.

Fonseca, M. P. 1999. Aphyllophorales lignocelulolíticos da Reserva Biológica do Alto da Serra de Paranapiacaba. $\mathrm{PhD}$ Thesis, Universidade de São Paulo, Brasil, 292pp.

Gaston, K. J. 2000. Global patterns in biodiversity. Nature, 405: 220-227. 
Gerber, A. L: Neves, M. A.; Loguercio-Leite, C. 1999. Some species of Perenniporia Murrill (Poriales, Basidiomycotina), from southern Brazil. Revista Brasileira de Botânica, 22: 185-193.

Gerber, A. L.; Loguercio-Leite, C. 1997. New records of polypores (Aphyllophorales) from southern Brazil. Mycotaxon, 62: 305-318.

Gerber, A. L.; Loguercio-Leite, C. 2000. Polyporoid wood-rotting fungi (Basidiomycetes) II - New records from southern Brazil. Mycotaxon, 76: 175-185.

Gilbert, G. S.; Ferrer, A.; Carranza, J. 2003. Polypore fungal diversity and host density in a moist tropical forest. Biodiversity and Conservation, 11: 947-957.

Gilbertoni, T.; Cavalcanti, M. A. Q. 2003. A mycological survey of the Aphyllophorales (Basidiomycotina) of the Atlantic Rain Forest in the sate of Pernambuco, Brazil. Mycotaxon, 89: 203-211.

Gilbertoni T.; Ryvarden, L.; Cavalcanti, M. A. Q. 2004. Poroid fungi (Basidiomycota) of the Atlantic rain forest in Northeast Brazil. Synopsis Fungorum, 18: 33-43.

Gilbertson, R. L.; Ryvarden, L. 1986. North America polypores Abortiporus - Lindtneria. Fungiflora, Oslo, Norway, 433pp.

Gilbertson, R. L.; Ryvarden, L. 1987. North America polypores Megasporosporia - Wrightoporia. Fungiflora, Oslo, Norway, $451 \mathrm{pp}$.

Góes-Neto, A. 1999. Polypore diversity in the state of Bahia, Brazil: a historical review. Mycotaxon, 72: 43-56.

Gonçalves G. V. C.; Loguercio-Leite, C. 2001; Biodiversidade de fungos poróides xilófilos (Basidiomycetes) na Unidade de Conservação Desterro (UCAD), Ilha de Santa Catarina, Brasil. Insula, 30: 01-19.

Groposo, C.; Loguercio-Leite, C. 2002. Fungos poliporóides xilófilos (Basidiomycetes) da Reserva Biológica Tancredo Neves, Cachoeirinha, Rio Grande do Sul, Brasil. Iheringia, 57: 39-59.

Groposo, C; Loguercio-Leite, C. 2004. Contribution to the lignocellulolytic fungi (Basidiomycetes) of the Atlantic Rain Forest in Southern Brazil. Mycotaxon, 92: 103-106.

Guerrero, R. T.; Homrich, M. H. 1999. Fungos macroscópicos comuns no Rio Grande do Sul. $2^{\text {a }}$ ed. Editora da UFRGS, Porto Alegre, Brasil, 124pp.

Gugliotta A. M.; Capelari, M. 1995. Polyporaceae from Ilha do Cardoso, SP, Brazil. Mycotaxon, 56: 107-113.

Holmgren, P. K.; Holmgren, N. H.; Barnett, L. C. 1990. Index Herbariorum. Part 1. The Herbaria of the World. International Association for Plant Taxonomy, Botanical Garden, $86^{\text {th }}$ ed. Bronx, New York, USA, 693pp.

Jesus, M. A. 1996. Contribution to the knowledge of wood-rotting fungi in Brazil. II. Check-list of fungi from Maracá Island, Roraima State. Mycotaxon, 57: 323-328.

Kirk, P. M.; Cannon, P. F.; David, J. C.; Stalpers, J. 2001. Ainsworth $\&$ Bisby's Dictionary of the Fungi. $9^{\text {th }}$ ed. Wallingford, CAB International, Oxon, USA, 655pp.

Larsen, M. J.; Cobb-Poulle, L. A. 1990. Phellinus (Hymenochaetaceae): A survey of the World Taxa. Fungiflora, Oslo, Norway, 206pp.

Loguercio-Leite, C.; Wright, J. E. 1991. New South American Pileate Polypores (Polyporaceae) from Santa Catarina Island, SC, Brazil. Mycotaxon, 41: 167-172.
Loguercio-Leite, C.; Wright, J. E. 1995. The Genus Phellinus (Hymenochaetaceae) on the Island of Santa Catarina, Brazil. Mycotaxon, 54: 361-388.

Myers, N. 1997. Florestas tropicais e suas espécies. In: Wilson, E. O. (ed.). Biodiversidade. Nova Fronteira, Rio de Janeiro, Brasil, p.36-45.

Rajchenberg, M.; Meijer, A. A. R. 1990. New and noteworthy polypores from Paraná and São Paulo Sates, Brazil. Mycotaxon, 38: 173-185.

Ryvarden, L. 1984. Type studies in the Polyporaceae 16. Species described by J. M. Berkeley, either alone or with other mycologists from 1856 to 1886. Mycotaxon, 20: 329-363.

Ryvarden, L. 1987. New and noteworthy polypores from tropical America. Mycotaxon, 28: 525-542.

Ryvarden, L. 2004. Neotropical Polypores. Part 1. Fungiflora, Oslo, Norway, 229pp.

Ryvarden, L.; Johansen, I. 1980. A preliminary polypore flora of East Africa. Fungiflora, Oslo, Norway, 636pp.

Ryvarden, L.; Meijer, A. A. R. 2002. Studies in neotropical polypores 14. New species from the State of Paraná, Brazil. Synopsis Fungorum, 15: 34-69.

Ryvarden L.; Wright J. E; Rajchenberg M. 1982. Megasporoporia, a new genus of resupinate polypores. Mycotaxon, 16: 172-182.

Ryvarden, L.; Meijer, A.A.R. 2002. Studies in neotropical polypores 14. New species from the State of Paraná, Brazil. Synopsis Fungorum, 15: 34-69.

Silveira, R. M. B.; Guerrero R. T. 1991. Aphyllophorales poliporóides (Basidiomycetes) do Parque Nacional de Aparados da Serra, Rio Grande do Sul. Boletim do Instituto de Biociências, 48: $1-127$.

Singer, R. 1975. The Agaricales in modern taxonomy. $3^{\text {th }}$ ed. Vaduz - J. Cramer, Stuttgart, Germany, 912pp.

Sippola, A. L.; Lehesvirta, T.; Renvall, P. 2001. Effects of selective logging on coarse woody debris and diversity of wood-decaying polypores in eastern Finland. Ecological Bulletins, 49: 243-254.

Soares, S. C. S.; Gugliotta, A. M. 1998. Criptógamos do Parque Estadual das Fontes do Ipiranga, São Paulo, SP. Fungos, 7: Aphyllophorales (Hymenochaetaceae). Hoehnea, 25 (1): 11-31.

Sótão H. M. P.; Campos, E. L.; Gugliotta, A. M.; Costa, S. P. S. E. 2003. Fungos Macroscópicos: Basidiomycetes. In: Fernandes, M. E. B. (org.). Os manguezais da costa norte brasileira. Fundação Rio Bacanga, São Luís, Brasil, p.45-59.

Suryanarayanan, T. S.; Venkatesan, G.; Murali, T. S. 2003. Endophytic fungal communities in leaves of tropical forest trees: diversity and distribution patterns. Current Science, 85: 489-493.

Wilson, E. O. 1997. A situação da diversidade biológica. In: Wilson, E. O. (ed.). Biodiversidade. Nova Fronteira, Rio de Janeiro, Brasil, p.3-26.

Zambonim, R. M. 2001. Banco de dados como subsídio para conservação e restauração nas tipologias vegetacionais do Parque Estadual da Serra do Tabuleiro e entorno. Dissertação de Mestrado, Universidade Federal de Santa Catarina, Florianópolis, Brasil, 217pp. 